\title{
Cutting-edge issues in core-collapse supernova theory
}

\author{
Kei Kotake ${ }^{1,2}$ \\ ${ }^{1}$ Division of Theoretical Astronomy, National Astronomical Observatory of Japan, 2-21-1, \\ Osawa, Mitaka, Tokyo, 181-8588, Japan \\ ${ }^{2}$ Center for Computational Astrophysics, National Astronomical Observatory of Japan, 2-21-1, \\ Osawa, Mitaka, Tokyo, 181-8588, Japan
}

\begin{abstract}
Based on our multi-dimensional neutrino-radiation hydrodynamic simulations, we report several cutting-edge issues about the long-veiled explosion mechanism of core-collapse supernovae (CCSNe). In this contribution, we pay particular attention to whether threedimensional (3D) hydrodynamics and/or general relativity (GR) would or would not help the onset of explosions. Our results from the first generation of full GR 3D simulations including approximate neutrino transport are quite optimistic, indicating that both of the two ingredients can foster neutrino-driven explosions. We give an outlook with a summary of the most urgent tasks to draw a robust conclusion to our findings.
\end{abstract}

Keywords. supernovae: general, hydrodynamics, relativity

\section{Introduction}

Ever since the first numerical simulation (Colgate \& White 1966), the neutrino-heating mechanism of CCSNe, in which a stalled bounce shock could be revived via neutrino absorption on a timescale of several hundred milliseconds after bounce, has been the working hypothesis of supernova theorists for $\sim 45$ years. However, the simplest, sphericallysymmetric (1D) form of this mechanism fails to blow up canonical massive stars (e.g., Liebendörfer et al. 2005). Pushed by mounting supernova observations of the blast morphology (e.g., Wang \& Wheeler 2008), it is now almost certain that the breaking of the spherical symmetry holds the key to solve the supernova problem (e.g., Kotake et al. 2006, Janka et al. 2007 for reviews). So far a number of multidimensional (multi-D) hydrodynamic simulations have shown that hydrodynamic motions associated with convective overturn (e.g., Herant et al. 1994) and the Standing-Accretion-Shock-Instability (SASI; e.g., Blondin et al. 2003, Ohnishi et al. 2006, Foglizzo et al. 2006, Iwakami et al. 2008, and references therein) can help the onset of the neutrino-driven explosion.

In fact, the neutrino-driven explosions have been obtained in the following firstprinciple two-dimensional (2D) simulations in which the spectral neutrino transport is solved at various levels of approximations (e.g., table 1 in Kotake 2011 for a summary). Among them are the 2D neutrino-radiation-hydrodynamic simulations by Buras et al. (2006) and Marek \& Janka (2009) who included one of the best available neutrino transfer approximations by the ray-by-ray variable Eddington factor method, by Bruenn et al. (2009) who included a ray-by-ray multi-group flux-limited diffusion transport with the best available weak interactions, and by Suwa et al. (2010) who employed a ray-by-ray isotropic diffusion source approximation (IDSA; Liebendörfer et al. 2009) with a reduced set of weak interactions.

This success, however, is opening further new questions. First of all, the explosion energies obtained in these $2 \mathrm{D}$ simulations are typically underpowered by one or two 
orders of magnitudes to explain the canonical supernova kinetic energy $\left(\sim 10^{51} \mathrm{erg}\right)$. Moreover, the softer nuclear equation of state (EOS), such as of Lattimer \& Swesty EOS with an incompressibility at nuclear densities, $K$, of $180 \mathrm{MeV}$, is employed in those simulations. On top of the striking evidence that favors a stiffer EOS based on the nuclear experimental data $(K=240 \pm 20 \mathrm{MeV})$, the soft EOS may not account for the recently observed massive neutron star of $\sim 2 M_{\odot}$ (Demorest et al. 2010). Using a stiffer EOS, the explosion energy may be even lower as inferred from Marek \& Janka (2009) who did not obtain the neutrino-driven explosion for their model with $K=263$ $\mathrm{MeV} \dagger$. What else is then missing? The neutrino-driven mechanism would be assisted by other candidate mechanisms such as the acoustic mechanism (Burrows et al. 2006) or the magnetohydrodynamic (MHD) mechanism (e.g., Takiwaki \& Kotake 2011 for collective references therein).

But before seeking alternative scenarios, it may be of primary importance to investigate how the explosion criteria extensively studied so far in 2D simulations could or could not be changed in $3 \mathrm{D}$ simulations. In section 2, we summarize our recent findings obtained in our 3D Newtonian simulations with spectral neutrino transport. In section 3, we move on to report our more recent results based on fully GR simulations including a more approximative neutrino transport.

\section{3D Newtonian simulations with spectral neutrino transport}

Going up the ladder beyond previous simulations (Nordhaus et al. 2010, Hanke et al. 2011) and using a light-bulb scheme to trigger parametric 3D explosions, we reported the first 3D, multigroup (via the IDSA scheme), radiation-hydrodynamic core-collapse simulations for an $11.2 M_{\odot}$ progenitor (Takiwaki et al. 2012). Firstly we briefly summarize the main results in this section.
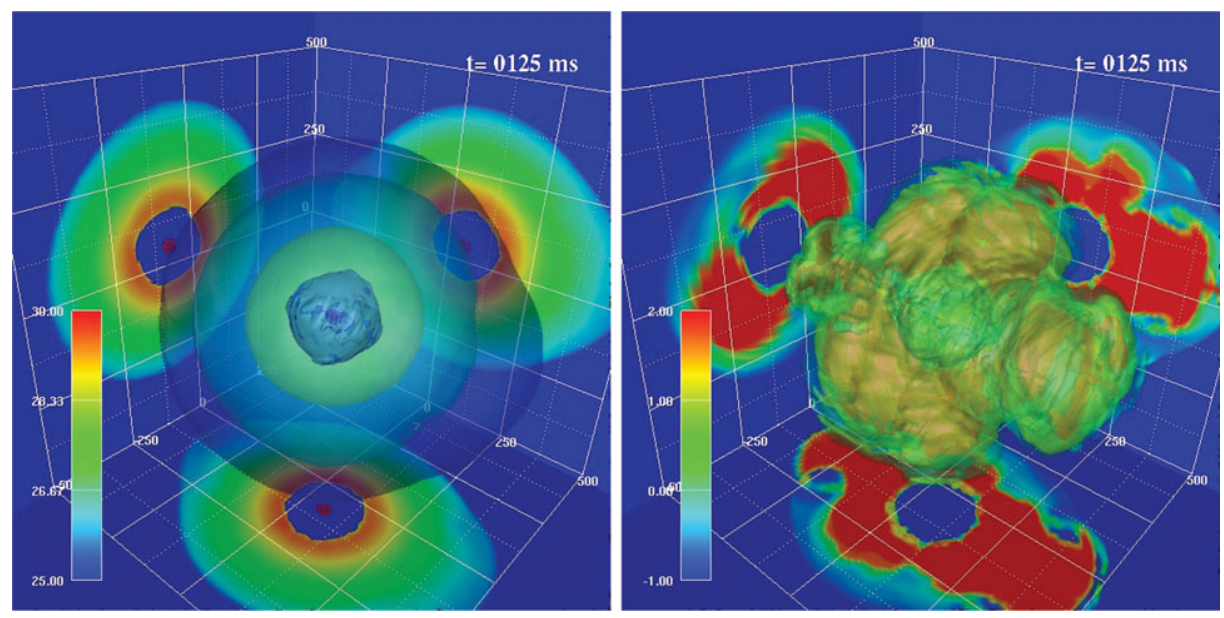

Figure 1. Left panel shows a snapshot for the net neutrino heating rate (logarithmic in unit of $\mathrm{erg} / \mathrm{cm}^{3} / \mathrm{s}$ at $t=125 \mathrm{~ms}$ after bounce) for a non-rotating $11.2 M_{\odot}$ star (Woosley et al. 2002). The right panel shows the ratio of the advection to the neutrino heating timescale.

Figure 1 shows snapshots for the net neutrino heating rate (left panel) and $\tau_{\text {res }} / \tau_{\text {heat }}$ :the ratio of the residency timescale to the neutrino-heating timescale (right panel) for a

$\dagger$ On the other hand, they obtained 2D explosions for Shen EOS $(K=281 \mathrm{MeV}$, H.-T. Janka, private communication). 
non-rotating $11.2 M_{\odot}$ star (Woosley et al. 2002; at $t=125 \mathrm{~ms}$ after bounce). The left panel shows that there forms the so-called gain region (seen as reddish regions in the wall panels). As shown in the right panel, the condition of $\tau_{\text {adv }} / \tau_{\text {heat }} \gtrsim 1$ is satisfied behind the aspherical shock, the low-mode deformation of which is characterized by the SASI. The time-scale ratio reaches about two in the gain region, which presents evidence that the shock-revival is driven by the neutrino-heating mechanism.
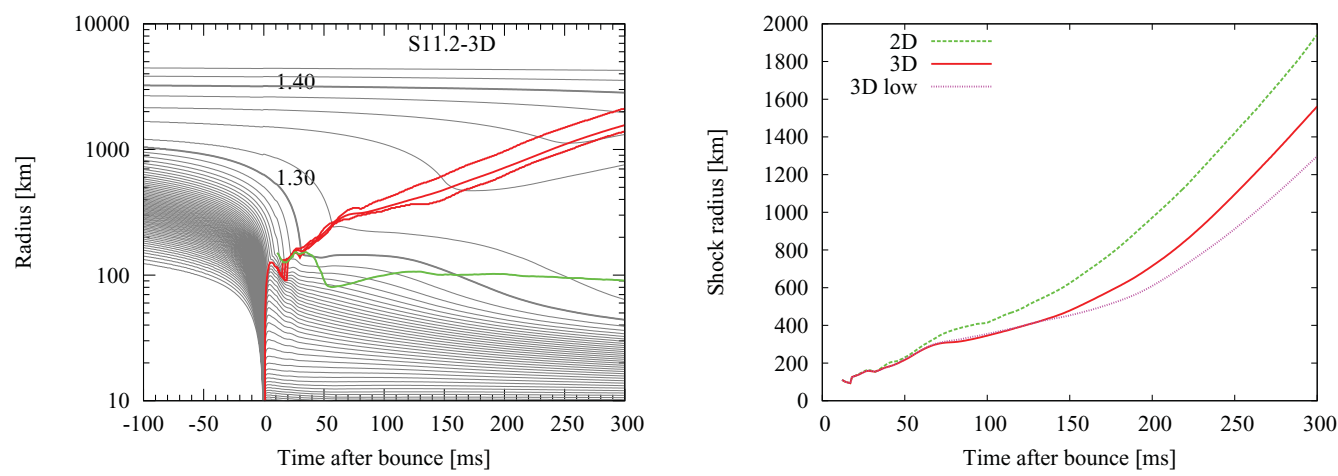

Figure 2. Time evolution of our $3 \mathrm{D}$ model of the $11.2 M_{\odot}$ star, visualized by mass shell trajectories in thin gray lines (left panel). Thick red lines show the position of shock waves, noting that the maximum (top), average (middle), and the minimum (bottom) shock position are shown, respectively. The green line represents the shock position of the 1D model. "1.30" and "1.40" indicates the mass in unit of $M_{\odot}$ enclosed inside the mass-shell. Right panel shows the evolution of average shock radii for the 2D (green line), and 3D (red line) models. The "3D low" (pink line) corresponds to the low resolution 3D model, in which the mesh numbers are taken to be half of the standard model (see text).

The left panel of Figure 2 show comparisons of the mass-shell trajectories between the 3D (red lines) and the corresponding 1D model (green line), respectively. At around 300 ms after bounce, the average shock radius for the $3 \mathrm{D}$ model exceeds $1000 \mathrm{~km}$ in radius. On the other hand, an explosion is not obtained for the 1D model. The right panel of Figure 2 shows a comparison of the average shock radius vs. postbounce time. In the 2D model, the shock expands rather continuously after bounce. These trends in 1D and 2D models are qualitatively consistent with Buras et al. (2006) (see, Takiwaki et al. 2012 for more detailed comparison).

Comparing the shock evolution between our 2D (green line in the right panel of Figure 2) and 3D model (red line), the shock is shown to expand much faster for 2D. The pink line labeled by "3D low" is for the low resolution 3D model, in which the mesh numbers are taken to be half of the standard model. Note that the 3D computational grid consists of 300 logarithmically spaced, radial zones to cover from the center up to $5000 \mathrm{~km}$ and 64 polar $(\theta)$ and 32 azimuthal $(\phi)$ uniform mesh points, which are used to cover the whole solid angle. The low resolution 3D model has one-half of the mesh numbers in the $\phi$ direction $\left(n_{\phi}=16\right)$, while fixing the mesh numbers in other directions. Comparing with our standard 3D model (red line), the shock expansion becomes less energetic for the low resolution model (later than $\sim 150 \mathrm{~ms}$ ). The above results indicate that explosions are easiest to obtain in 2D, followed in order by 3D, and 3D (low). At first sight, this may look like a contradiction with the findings obtained in parametric 3D explosion models (e.g., Nordhaus et al. 2010) which pointed out that explosions could be more easily obtained in $3 \mathrm{D}$ than in $2 \mathrm{D}$. The reason for the discrepancy is summarized shortly as follows.

Figure 3 compares the blast morphology for our 3D (left panel) and 2D (right) model. In the 3D model (left panel), non-axisymmetric structures are clearly seen. By performing 
a tracer-particle analysis, the maximum residency time of material in the gain region is shown to be longer for 3D than $2 \mathrm{D}$ due to the non-axisymmetric flow motions. This is one of advantageous aspects of 3D models to obtain neutrino-driven explosions. On the other hand, our detailed analysis showed that convective matter motions below the gain radius become much more violent in $3 \mathrm{D}$ than in $2 \mathrm{D}$, making the neutrino luminosity larger for 3D (e.g., Takiwaki et al. 2011). Nevertheless the emitted neutrino energies are made smaller due to the enhanced cooling. Due to these competing ingredients, the neutrino heating timescale becomes shorter for the 3D model, leading to a smaller netheating rate compared to the corresponding $2 \mathrm{D}$ model. Note here that the spectral IDSA scheme, by which the feedback between the mass accretion and the neutrino luminosity can be treated in a self-consistent manner (not like the light-bulb scheme assuming a constant luminosity), sounds quite efficient in the first-generation 3D simulations.

As seen from Figure 2, an encouraging finding is that the shock expansion tends to become more energetic for models with finer resolutions. These results would indicate whether these advantages for driving 3D explosions can or cannot overwhelm the disadvantages in sensitivity to the employed numerical resolutions $\dagger$. To draw a robust conclusion, 3D simulations with much higher numerical resolutions and more advanced treatment of neutrino transport as well as of gravity are needed, which hopefully will be practical by utilizing forthcoming petaflops-class supercomputers.
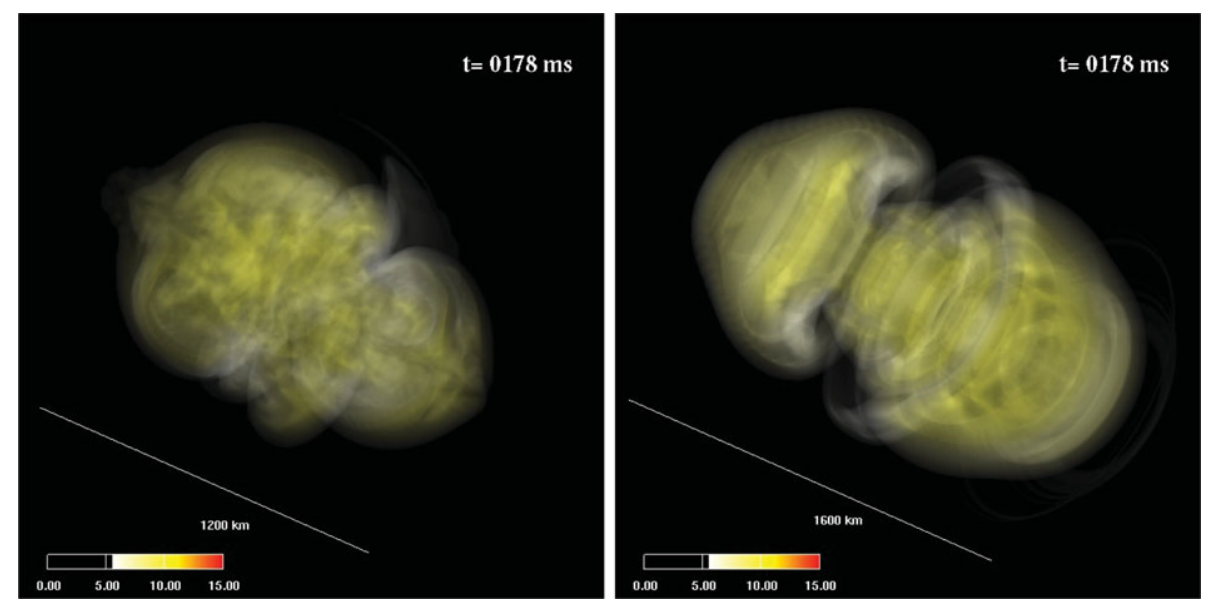

Figure 3. Volume rendering of entropy showing the blast morphology in our 3D (left) and 2D (right) model for the $11.2 M_{\odot}$ progenitor (at $t=178 \mathrm{~ms}$ after bounce), respectively. In both panels, the polar axis is tilted (about $\pi / 4$ ). The linear scale is indicated in each panel.

\section{3D full GR simulations with an approximate neutrino transport}

In addition to the 3D effects, impacts of GR on the neutrino-driven mechanism stand out among the biggest open questions in supernova theory. Since the late 1990s, the ultimate 1D simulations, in which the GR Boltzmann transport is coupled to 1D GR hydrodynamics, have been made feasible (e.g., Sumiyoshi et al. 2005, Liebendörfer et al. 2004, and references therein). In these full-fledged 1D simulations, a commonly observed disadvantageous aspect of GR to drive neutrino-driven explosions is that the residency time of material in the gain region becomes shorter due to the stronger gravitational pull.

$\dagger$ It is of crucial importance to conduct a convergence test in which a numerical gridding is changed in a systematic way (e.g. Hanke et al. 2011). 
In $1 \mathrm{D}$, it is generally agreed that GR works disadvantageously to facilitate the neutrinodriven explosions (e.g., Lentz et al. 2011). Although extensive attempts have been made to include microphysics such as by the $Y_{e}$ formula or by a neutrino leakage scheme in multi-D GR simulations, the effects of neutrino heating have yet to be included in them, which is a main hindrance to studying the GR effects on the multi-D neutrino-driven mechanism $\ddagger$.

Here we present the first 3D simulations in full GR that include an approximate treatment of neutrino transport (Kuroda et al. 2012). The code is a marriage of an adaptivemesh-refinement (AMR), conservative 3D GR MHD code developed by Kuroda \& Umeda (2010), and the approximate neutrino transport code that we recently developed in this work. The spacetime treatment in our full GR code is based on the Baumgarte-ShapiroShibata-Nakamura formalism. The hydrodynamics can be solved either in full GR or in special relativity (SR), which allows us to investigate the GR effects on the supernova dynamics. Using a M1 closure scheme with an analytic variable Eddington factor, we solve the energy-independent set of radiation energy and momentum. This part is based on the partial implementation of the Thorne's momentum formalism (Thorne 1981), which has recently been extended by Shibata et al. (2011) in a more suitable manner applicable to the neutrino transport problem (see Kuroda et al. 2012 for more details).

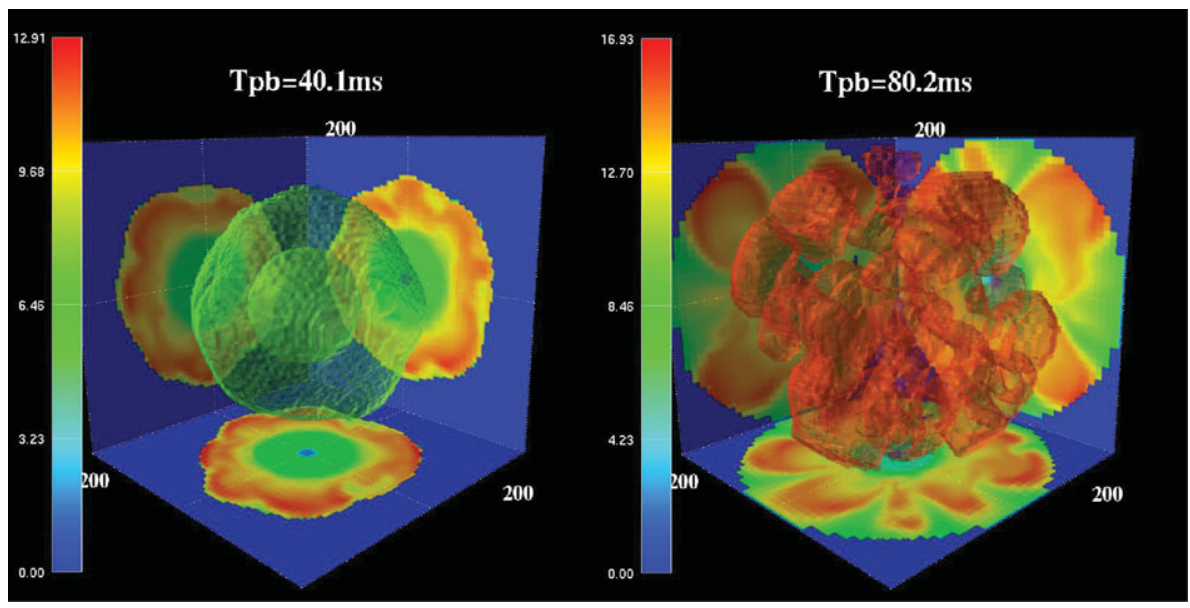

Figure 4. Three dimensional plots of entropy per baryon for four snapshots (left; $t_{\mathrm{pb}}=40 \mathrm{~ms}$ and right; $t_{\mathrm{pb}}=100 \mathrm{~ms}$ ) for our $3 \mathrm{D}-\mathrm{GR}$ model of a $15 M_{\odot}$ star. The contours on the cross sections in the $x=0$ (back right), $y=0$ (back bottom), and $z=0$ (back left) planes are, respectively projected on the sidewalls of the graphs to visualize 3D structures. For each snapshot, the arbitrarily chosen iso-entropy surface is shown, and the linear scale is indicated along the axis in units of $\mathrm{km}$.

The left panel of Figure 4 shows a snapshot at $t=40 \mathrm{~ms}$ postbounce for our 3D GR model $\dagger$. At this stage, the bounce shock stalls (seen as a greenish sphere) and the gain region forms at $\sim 80 \mathrm{~ms}$ after bounce in which neutrino heating dominates over neutrino cooling. The neutrino-driven convection gradually develops later on. The entropy

$\ddagger$ Very recently, Müller et al. (2012) reported explosions for 11.2 and $15 M_{\odot}$ stars based on their 2D GR simulations in CFC with detailed neutrino transport similar to Buras et al. (2006).

$\dagger$ The 3D computational domain consists of a cube of $10000^{3} \mathrm{~km}^{3}$ volume in Cartesian coordinates. The maximum refinement AMR level is 5 in the beginning and then increments as the collapse proceeds. The criterion to increment $L_{\mathrm{AMR}}$ is set every time the central density exceeds $10^{12,13,13.5} \mathrm{~g} \mathrm{~cm}^{-3}$ (see Kuroda \& Umeda 2010 for more details), yielding an effective resolution of $\Delta x \sim 600 \mathrm{~m}$ at bounce. 
behind the standing shock becomes higher with time due to neutrino-heating. The high entropy bubbles $\left(s\left[k_{B} /\right.\right.$ baryon $\left.] \gtrsim 10\right)$ rise and sink behind the standing shock. The shock deformation is dominated by unipolar and bipolar modes, which is a characteristic feature of the SASI. The size of the neutrino-heated regions grows bigger with time in a non-axisymmetric way, which is indicated by bubbly structures with increasing entropy (indicated by reddish regions in the right panel).

During our simulation time (100 ms after bounce), the shock radii can reach further out for our 3D-GR model. In contrast, the shock has already shown a trend of recession in other models, namely for 1D-SR, 1D-GR, 3D-SR models, in which "SR" stands for special relativity.

The left panel of Figure 5 shows evolution of the neutrino luminosities (for $\nu_{e}$ and $\nu_{x}$ ) for all the computed models. After the neutronization burst $\left(t_{\mathrm{pb}} \sim 10 \mathrm{~ms}\right)$, the $\nu_{e}$ luminosity for the GR models slightly increases later on, while it stays almost constant for the SR models during the simulation time (green and blue lines). Although the luminosities change with time, the luminosities generally yield to the following order,

for $\nu_{e}, 3 \mathrm{D}-\mathrm{GR}>1 \mathrm{DGR}, 3 \mathrm{D}-\mathrm{SR} \sim 1 \mathrm{D}-\mathrm{SR}$,

for $\bar{\nu}_{e}, 3 \mathrm{D}-\mathrm{GR}>1 \mathrm{DGR}, 3 \mathrm{D}-\mathrm{SR}>1 \mathrm{D}-\mathrm{SR}$,

for $\nu_{x}, 3 \mathrm{D}-\mathrm{GR}>1 \mathrm{DGR}, 3 \mathrm{D}-\mathrm{SR}>1 \mathrm{D}-\mathrm{SR}$.

To summarize, both $3 \mathrm{D}$ and GR work to raise the neutrino luminosities in the early postbounce phase. As seen from the left panel in Figure 5, GR maximally increases the $\nu_{x}$ luminosity up to $\sim 50 \%$ (in 3D), while the maximum increase by $3 \mathrm{D}$ is less than $\sim 20 \%$ (compare the $\bar{\nu}_{e}$ luminosity between the 3D-GR and 1D-GR model). These results indicate that compared to the spacial dimensionality, GR holds the key to enhancing the neutrino luminosities. This is also the case for the RMS neutrino energy. The reason for the higher neutrino energy is that the deeper gravitational well of GR produces more compact core structures, and thus hotter neutrino spheres at smaller radii.
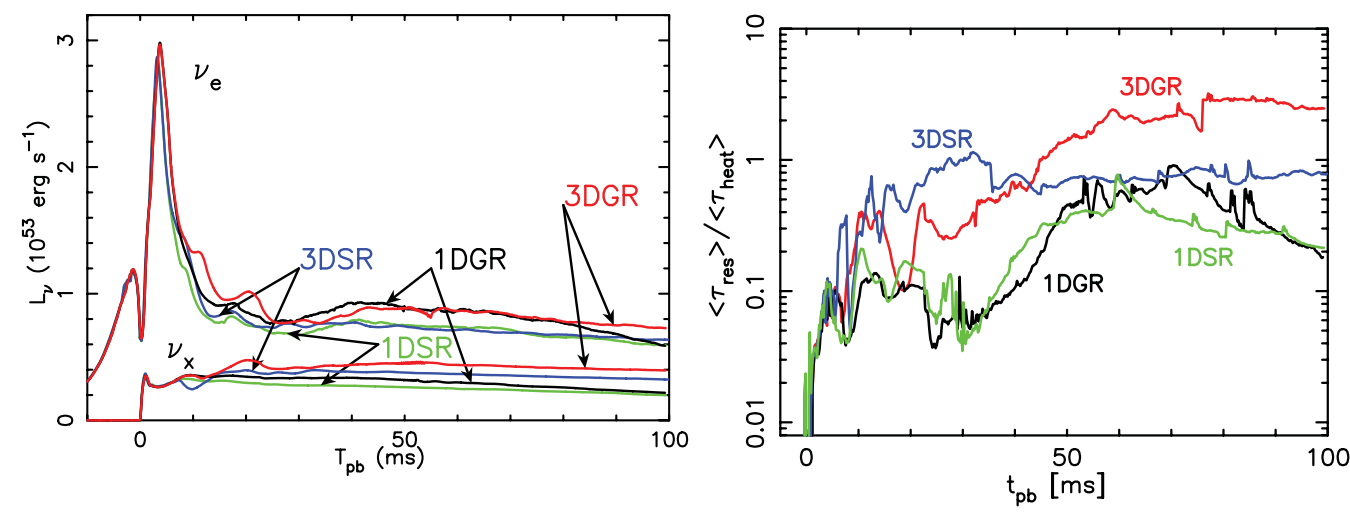

Figure 5. Neutrino luminosities for $\nu_{e}, \nu_{x}$ (left panel) as a function of postbounce time, respectively. Right panel shows the ratio of the residency timescale to the heating timescale for the set of our models as functions of post-bounce time (see Kuroda et al. 2012 for the definition of the timescales).

The right panel of Figure 5 shows the ratio of the residency timescale to the neutrinoheating timescale for all the computed models. As seen, the shock revival seems most likely to occur for the 3D-GR model (red line) in our simulation time, which is followed in order by 3D-SR, 1D-SR and 1D-GR models. Thanks to more degrees of freedom, the residency timescale becomes much longer for the $3 \mathrm{D}$ models than for the $1 \mathrm{D}$ models. In addition, the increase of the neutrino luminosity and RMS energies due to GR enhances 
the timescale ratio up to the factor of $\sim 2$ for the 3D-GR model (red line) compared to the SR counterpart (blue line). Therefore our results suggest that the combination of $3 \mathrm{D}$ and GR hydrodynamics could provide the most favorable condition to trigger the neutrino-driven explosions.

For the $15 M_{\odot}$ progenitor employed in our GR simulation, the neutrino-driven explosions are expected to take place later than $\sim 200 \mathrm{~ms}$ postbounce at the earliest (Bruenn et al. 2009) and it could be delayed after $\sim 600 \mathrm{~ms}$ postbounce (Marek \& Janka 2009). The parametric explosion models have shown that the earlier shock revival is good for making the explosion energy larger (e.g., Nordhaus et al. 2010). The onset timescale of the neutrino-driven explosions predicted in 2D models (Marek \& Janka 2009, Bruenn et al. 2009, Suwa et al. 2010) could be shorter if the combined effects of GR and 3D would have been included. We anticipate that this can be a possible remedy to turn the relatively underpowered $2 \mathrm{D}$ explosions into the powerful ones. It is worth mentioning that the combined effects of GR and 3D should affect not only the supernova dynamics, but also the observational signatures of gravitational-waves (e.g., Kotake et al. 2011), neutrino emission (e.g., Abbasi et al. 2011), and explosive nucleosynthesis (e.g., Fujimoto et al. 2011). To draw a solid conclusion to these important observables, the energy and angle dependence of neutrino transport should be accurately incorporated in our full GR simulations with the use of a more detailed set of weak interactions. This work is only the very first step that leads us to the long and winding road.

Finally, it should be noted that all the numerical results presented in this article should be tested by the next-generation calculations by which more sophistication is made not only in the treatment of multi-D neutrino transport but also in multi-D hydrodynamics including stellar rotation and magnetic fields in full GR. From an optimistic point of view, our understanding of the explosion mechanism can progress in a step-by-step manner at the same pace as our available computational resources will be growing bigger and bigger from now on. Since 2009, several neutrino detectors form the Supernova Early Warning Systems (SNEWS) to broadcast alerts to astronomers to let them know the arrival of neutrinos (Antonioli et al. 2004). Currently, Super-Kamiokande, LVD, Borexino, and IceCube contribute to the SNEWS, with a number of other neutrino and GW detectors planning to join in the near future. This is very encouraging news for high-precision multimessenger astronomy. The interplay between detailed numerical modeling, advancing supercomputing resources, and multi-messenger astronomy, will remain a central issue for advancing our understanding of the theory of massive stellar core-collapse for the future.

\section{References}

Abbasi, R., et al. 2011, A\&GA, 535, A109

Alcubierre, M. \& Brügmann, B. 2001, Phys. Rev. D., 63, 104006

Antonioli, P., et al. 2004, New Journal of Physics, 6, 114

Blondin, J. M., Mezzacappa, A., \& DeMarino, C. 2003, ApJ, 584, 971

Bruenn, S. W., Mezzacappa, A., Hix, W. R., Blondin, J. M., Marronetti, P., Messer, O. E. B., Dirk, C. J., \& Yoshida, S. 2010, arXiv:1002.4909

Buras, R., Rampp, M., Janka, H.-Th., \& Kifonidis, K. 2006, A\&A, 447, 1049

Burrows, A., Livne, E., Dessart, L., Ott, C. D., \& Murphy, J. 2006, ApJ, 640, 878

Colgate, S. A. \& White, R. H. 1966, ApJ, 143, 626

Demorest, P. B., Pennucci, T., Ransom, S. M., Roberts, M. S. E., \& Hessels, J. W. T. 2010, Nature, 467,1081

Foglizzo, T., Scheck, L., \& Janka, H.-T. 2006, ApJ, 652, 1436

Fujimoto, S.-I., Kotake, K., Hashimoto, M.-A., Ono, M., \& Ohnishi, N. 2011, ApJ, 738, 61 
Hanke, F., Marek, A., Mueller, B., \& Janka, H.-T. 2011, arXiv:1108.4355

Herant, M., Benz, W., Hix, W. R., Fryer, C. L., \& Colgate, S. A. 1994, ApJ, 435, 339

Iwakami, W., Kotake, K., Ohnishi, N., Yamada, S., \& Sawada, K. 2008, ApJ, 678, 1207

Janka, H. \& Müller, E. 1996, A\&̊A, 306, 167

Janka, H.-T., Langanke, K., Marek, A., Martínez-Pinedo, G., \& Müller, B. 2007, Phy. Rep., 442, 38

Kotake, K. 2011, Comptes Rendus Physique, accepted (arXiv:1110.5107)

Kotake, K., Sato, K., \& Takahashi, K. 2006, Reports of Progress in Physics, 69, 971

Kotake, K., Iwakami-Nakano, W., \& Ohnishi, N. 2011, ApJ, 736, 124

Kuroda, T. \& Umeda, H. 2010, ApJS, 191, 439

Kuroda, T., Kotake, K., \& Takiwaki, T. 2012, ApJ, submitted (arXiv:1202.2487)

Lentz, E. J., et al. 2011, arXiv:1112.3595

Liebendörfer, M., et al. 2004, ApJS, 150, 263

Liebendörfer, M., Rampp, M., Janka, H.-T., \& Mezzacappa, A. 2005, ApJ, 620, 840

Liebendörfer, M., Whitehouse, S. C., \& Fischer, T. 2009, ApJ, 698, 1174

Marek, A. \& Janka, H.-T. 2009, ApJ, 694, 664

Masada, Y., Takiwaki, T., \& Kotake, K. 2011, ApJ, submitted

Müller, B., Janka, H.-T., \& Marek, A. 2012, arXiv:1202.0815

Nordhaus, J., Burrows, A., Almgren, A., \& Bell, J. 2010, ApJ, 720, 694

Ohnishi, N., Kotake, K., \& Yamada, S. 2006, ApJ, 641, 1018

Shibata, M., Kiuchi, K., Sekiguchi, Y., \& Suwa, Y. 2011, Progress of Theoretical Physics, 125, 1255

Sumiyoshi, K., Yamada, S., Suzuki, H., Shen, H., Chiba, S., \& Toki, H. 2005, ApJ, 629, 922

Suwa, Y., Kotake, K., Takiwaki, T., Whitehouse, S. C., Liebendörfer, M., \& Sato, K. 2010, PASJ (Letters), 62, L49

Takiwaki, T. \& Kotake, K. 2011, ApJ, 743, 30

Takiwaki, T., Kotake, K., \& Suwa, Y. 2012, ApJ, 749, 98

Thorne, K. S. 1981, MNRAS, 194, 439

Wang, L. \& Wheeler, J. C. 2008, ARAA, 46, 433

Woosley, S. E., Heger, A., \& Weaver, T. A. 2002, Reviews of Modern Physics, 74, 1015

\section{Discussion}

KeIICHI Nishikawa: Could you explain about GR model? Do you include MHD?

KeI Kotake: The metric evolution is solved by the BSSN formalism with the HRSC scheme to evolve GR hydrodynamics. The code is already written in MHD, but we have only run non-magnetized models at present. As you would imply, MHD simulations in GR presumably in the context of collapsar should be very interesting.

SerGey MoiseEnKo: MHD mechanism of SN explosion gives explosion energy $10^{51} \mathrm{erg}$ which is enough for explaining the observed kinetic energy.

KEI KoTAKE: That's right, but only if the precollapse core has strong B-fields with rapid rotation. But I agree with you that the MHD mechanism should be revisited by our GR (or more importantly) 3D models.

SeAn Couch: Have you tried any rotating models?

Kei Kotake: Not yet, but this is what we are currently undertaking. 